\title{
Avaliação da Restauração Florestal de uma APP Degradada em Santa Catarina
}

\author{
Carla Carolina Chini Rech ${ }^{1}$, Ana Carolina da Silva ${ }^{1}$, Pedro Higuchi ${ }^{1}$, \\ Marcos Benedito Schimalski ${ }^{1}$, Francieli Pscheidt ${ }^{1}$, Arthur Bratti Schmidt ${ }^{1}$, \\ Roni Djeison Ansolin ${ }^{1}$, Marco Antônio Bento ${ }^{1}$, \\ Francieli de Fátima Missio ${ }^{1}$, Rodineli Loebens ${ }^{1}$
}

${ }^{1}$ Departamento de Engenharia Florestal, Universidade do Estado de Santa Catarina - UDESC, Lages/SC, Brasil

\section{RESUMO}

Este trabalho objetivou avaliar o processo de restauração em uma Área de Preservação Permanente (APP) degradada em Pouso Redondo, SC, seis anos após o plantio de mudas e proteção da área. Foi realizado o levantamento da vegetação arbórea e, a partir da relação das espécies plantadas inicialmente, foi quantificado o aumento da riqueza que ocorreu por meio do estabelecimento natural de novas espécies. Foram determinadas as síndromes de dispersão de propágulos e as guildas de regeneração. Foram encontrados 918 indivíduos pertencentes a 73 espécies, sendo que $48(65,8 \%)$ não correspondem àquelas que foram plantadas em 2007, tratando-se de novas espécies que se estabeleceram. $71,2 \%$ das espécies foram classificadas como zoocóricas e 54,8\% como clímax exigente em luz. Após seis anos, o processo de restauração foi caracterizado pela tendência do aumento da riqueza e da substituição de espécies iniciais por tardias, indicando a relevância do mecanismo de regeneração natural.

Palavras-chave: regeneração natural, recuperação de áreas degradadas, sucessão florestal.

\section{Evaluation of Forest Restoration in a Degraded Permanent Preservation Area in Santa Catarina State, Brazil}

\begin{abstract}
This study evaluated the process of restoration in a degraded Permanent Preservation Area (PPA) in the municipality of Pouso Redondo, Santa Catarina state, six years after the initial plantation of trees and isolation of the area. Tree species were surveyed and increment of richness by natural establishment was quantified. Syndromes of propagule dispersion and regeneration guilds were determined. A total of 918 individuals from 73 species were found; 48 (65.8\%) of them were species naturally established in the area. $71.2 \%$ of the species were classified as zoochoric (71.2\%), and $54.8 \%$ as light demanding for climax. After six years, the restoration process was characterized by a trend of richness increment and replacement of initial by late successional species, indicating the relevance of the natural regeneration mechanism.
\end{abstract}

Keywords: natural regeneration, degraded area restoration, forest succession. 


\section{INTRODUÇÃO}

O processo de ocupação do território brasileiro foi caracterizado pela destruição dos recursos naturais, com a substituição de florestas por outros usos dos solos. As florestas ciliares, por estarem em locais estratégicos para a ocupação humana, são um dos ecossistemas mais perturbados e/ou degradados entre os ambientes florestais. Porém, devido à sua importância na manutenção da qualidade da água, estabilidade do solo, regularização do regime hídrico e manutenção de corredores para o movimento da fauna e dispersão vegetal (Alvarenga et al., 2006), essas áreas são consideradas Áreas de Preservação Permanente e são delimitadas faixas mínimas para a sua preservação. Portanto, considerando o atual estágio de degradação de grande parte dessas florestas, é importante a definição e avaliação de técnicas de restauração dessas áreas.

A aplicação de estratégias para a restauração florestal visa restabelecer os processos e a estrutura do ecossistema original, garantindo incrementos em biodiversidade nos ecossistemas degradados (Kageyama \& Gandara, 2005). Atualmente, diversas técnicas de restauração têm sido propostas visando maximizar os benefícios e acelerar o processo como, por exemplo, o plantio de mudas, o plantio de materiais vegetativos (estacas) diretamente no campo, a semeadura direta, a chuva de sementes, a colocação de poleiros artificiais, entre outros, e alguns estudos que visam avaliar técnicas ou métodos têm sido realizados (p. ex., Araujo et al., 2004; Reis et al., 2007; Rodrigues et al., 2007). A técnica adequada a cada área depende dos níveis de degradação e fonte de propágulos, sendo necessário o acompanhamento e a avaliação dos resultados da implantação das medidas de recuperação. No entanto, o monitoramento raramente é implementado de modo sistemático em razão da dificuldade de se estabelecerem parâmetros ou critérios para avaliação do desempenho da restauração (Almeida \& Sánchez, 2005), sendo poucas as avaliações a médio e longo prazo dessas áreas (p. ex., Valcarcel \& D’Altério, 1998; Durigan \& Silveira, 1999; Almeida \& Sánchez, 2005; Alvarenga et al., 2006; Santos \& Valcarcel, 2011), especialmente no estado de Santa Catarina (p. ex., Figueiredo, 2005).

Para a avaliação de áreas restauradas devem ser recolhidos parâmetros que forneçam uma informação agregada e sintética sobre algum aspecto da própria vegetação, do solo e da fauna, para que se possa verificar a eficiência da cobertura vegetal na proteção do solo, na produtividade dos ecossistemas ou mesmo sua função como refúgio da vida silvestre (Almeida \& Sánchez, 2005). Dessa forma, considerando-se a importância de realizar o monitoramento das áreas restauradas para identificar técnicas de recomposição mais eficientes, o presente estudo objetivou avaliar o processo de restauração ecológica em uma Área de Preservação Permanente (APP) degradada, no entorno de uma nascente e de um curso d'água, em Pouso Redondo, região do Alto Vale do Itajaí, SC, seis anos após o plantio de mudas e proteção integral da área.

\section{MATERIAL E MÉTODOS}

O estudo foi realizado em um trecho de APP, em área no entorno de nascentes e cursos de água, localizada no município de Pouso Redondo, SC (Figura 1). Pouso Redondo está localizado na microrregião de Rio do Sul, no Alto Vale da bacia hidrográfica do Rio Itajaí-Açú. A área de estudo está a $27^{\circ} 15^{\prime} 19^{\prime \prime}$ de latitude Sul e $49^{\circ} 53^{\prime} 03^{\prime \prime}$ de longitude Oeste, a uma altitude média de $385 \mathrm{~m}$. A região possui clima Cfa, segundo a classificação de Köppen, e a precipitação anual média, de acordo com os dados do WORLDCLIM (Hijmans et al., 2005) é de aproximadamente $1.499 \mathrm{~mm}$, com temperatura média anual de $18,9^{\circ} \mathrm{C}$. Na região do Alto Vale do Itajaí predomina uma floresta de transição entre Floresta Ombrófila Densa e Floresta Ombrófila Mista, segundo a classificação do IBGE (2012), formações vegetais pertencentes aos domínios da Mata Atlântica.

A área de estudo é adjacente a uma área de mata nativa (aproximadamente $40 \mathrm{~m}$ da fonte de propágulo) e, até o ano de 2007, a extensão do curso d'água estava desprotegida e sujeita ao impacto do gado, não havendo remanescentes de mata ciliar. $\mathrm{A}$ fim de adequar as áreas à legislação ambiental, no que se refere à proteção de cursos d'água, em dezembro de 2007 realizou-se a revegetação da área por meio do plantio de cerca de 1.500 mudas de 44 espécies em uma área de aproximadamente 1 ha. Para isso foi empregada a metodologia proposta pela equipe do Laboratório de Ecologia e Recuperação Florestal (LERF) da ESALQ/USP (Attanasio et al., 2006), na qual são plantados indivíduos pertencentes a espécies arbóreas de dois grupos: grupo de preenchimento (25\% de pioneiras e $25 \%$ de secundárias iniciais) e grupo de 


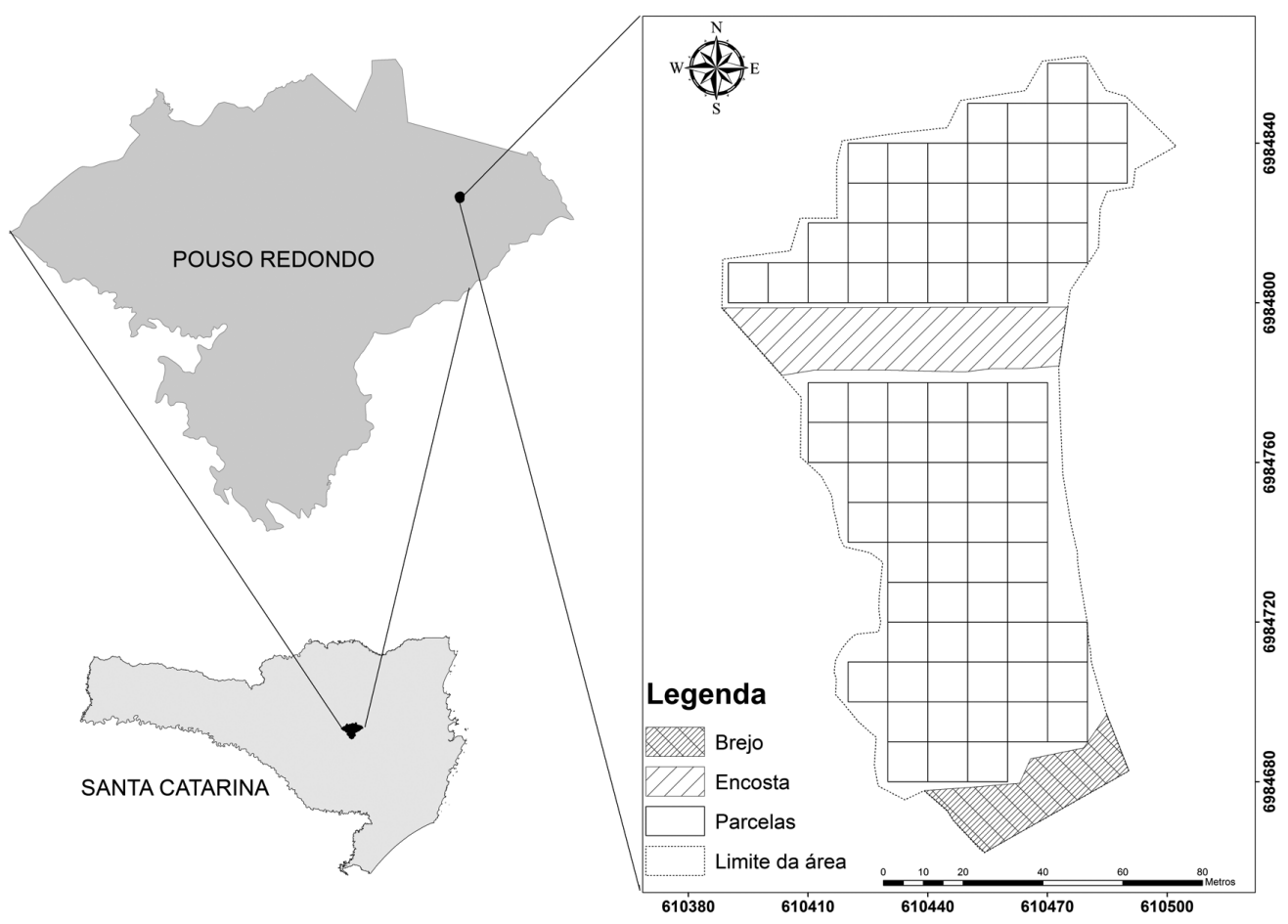

Figura 1. Localização da Área de Preservação Permanente (APP) em processo de restauração em Pouso Redondo, SC. Figure 1. Location of Permanent Preservation Area (APP) under restoration process, in the municipality of Pouso Redondo, SC.

diversidade (25\% de secundárias iniciais intercaladas com $25 \%$ de secundárias tardias e clímaces). Das 44 espécies implantadas na área, 33 foram do grupo de diversidade e 11, do grupo de preenchimento. Os grupos de plantio foram na forma de modelos de linhas alternadas, sendo uma linha de preenchimento e outra de diversidade, arranjadas nos espaçamentos de $3 \mathrm{~m}$ entre linhas e $2 \mathrm{~m}$ entre plantas na linha. Durante o plantio, foram abertas covas de aproximadamente $30 \times 30 \times 30 \mathrm{~cm}$, que receberam somente a terra do local, sem adubação. Um mês após o plantio, o proprietário realizou o coroamento das mudas. $\mathrm{O}$ controle de formigas foi feito pelo proprietário desde o plantio com uso do inseticida ORTHENE 750 BR nos formigueiros, no momento em que ele percebeu o surgimento dos insetos.

Para avaliação da recuperação, nos meses de fevereiro a agosto de 2013 foi realizado o levantamento dos indivíduos arbóreos plantados sobreviventes e os que se estabeleceram na área por meio de regeneração natural. Para isso, a área foi dividida em parcelas, totalizando 82 parcelas de $10 \times 10 \mathrm{~m}$ (0,82 ha) distribuídas de forma contínua. As áreas adjacentes de encosta e banhado (brejo), onde não houve o plantio de mudas, não foram inventariadas. Dentro das parcelas foi realizado o censo dos indivíduos arbóreos adultos (considerados aqueles com DAP - diâmetro medido a 1,30 m do solo - igual ou maior a $5 \mathrm{~cm}$ ), que tiveram sua identidade botânica registrada. Para o inventário dos indivíduos arbóreos jovens (considerados aqueles com mais de $1 \mathrm{~m}$ de altura e com menos de $5 \mathrm{~cm}$ de DAP), foi realizada uma amostragem, sendo que as 82 parcelas foram divididas em subparcelas de $5 \times 5 \mathrm{~m}\left(25 \mathrm{~m}^{2}\right)$, nas quais todos os indivíduos arbóreos jovens foram identificados. Quando não foi possível conhecer a identidade do indivíduo a campo, ele foi coletado e identificado por especialistas. As espécies foram classificadas em famílias de acordo com o sistema APG III (APG, 2009).

Foram determinadas a porcentagem das espécies plantadas sobreviventes e a porcentagem das espécies que se estabeleceram por regeneração natural. Foi quantificada a riqueza de espécies plantadas em 2007 e das inventariadas em 2013. Foram calculados os índices de diversidade de Shannon-Wiener $\left(\mathrm{H}^{\prime}\right)$ e a equabilidade de Pielou (J) (Brower \& Zar, 1984) das espécies amostradas em 2013. Os índices foram calculados 
para os indivíduos adultos e jovens utilizando-se o programa R (R Development Core Team, 2014).

As espécies foram classificadas de acordo com a síndrome de dispersão de propágulos, segundo a metodologia de Van der Pijl (1982), na qual: i) espécies de frutos carnosos e com outros elementos que evidenciam a dispersão por animais foram consideradas como de dispersão zoocórica; ii) frutos ou sementes com alas e mecanismos para flutuação foram definidas como anemocóricas; iii) frutos tipo cápsula deiscente que, ao se abrirem, liberam as sementes por um rápido movimento foram consideradas de dispersão autocórica. A determinação dessas síndromes se deu por observações dos diásporos e por consultas à literatura (Rondon Neto et al., 2001; Budke et al., 2005; Giehl et al., 2007; Almeida et al., 2008; Leyser et al., 2009; Negrini et al., 2012).

O estágio sucessional em que se encontra a floresta foi definido por meio da classificação das espécies em grupos sucessionais, utilizando-se a metodologia de Swaine \& Whitmore (1988), que divide as espécies em dois grandes grupos: pioneiras, aquelas que necessitam de luz para germinar e completar o ciclo de vida, e clímax, aquelas que frequentemente ocorrem no sub-bosque florestal. Conforme proposto por Oliveira Filho et al. (1994), o grupo das climácicas foi subdivido em função da exigência lumínica das espécies em "exigentes em luz", que são aquelas que precisam de luz para completar o ciclo de vida, e "tolerantes ao sombreamento", que são as que germinam em condição de sub-bosque e podem completar o ciclo de vida nessas condições. Para a classificação das espécies utilizou-se a literatura existente (p. ex., Lindenmaier \& Budke, 2006; Leyser et al., 2012) e observações no campo.

\section{RESULTADOS E DISCUSSÃO}

Foram inventariados 918 indivíduos arbóreos pertencentes a 73 espécies (Tabela 1). Dessas, três foram identificadas somente ao nível de gênero e uma apenas em nível de família botânica. Devido à ausência de folhas, 15 indivíduos não puderam ser identificados. Do total de indivíduos inventariados, 366 eram adultos e 552 jovens. Os 366 adultos pertenciam a 40 espécies, distribuídas em 34 gêneros e 20 famílias botânicas. Os 552 indivíduos jovens pertenciam a 67 espécies, distribuídas em 48 gêneros e 25 famílias botânicas.
Das 73 espécies encontradas no total, 48 (65,8\%) não correspondem àquelas que foram plantadas no ano de 2007, tratando-se, portanto, de novas espécies que se estabeleceram na área, seja por meio da dispersão de propágulos ou banco de sementes, o que contribuiu para o aumento da riqueza da área. Isso indica que a regeneração natural contribuiu de forma significativa para a riqueza atual, o que pode ser explicado, também, pela proximidade da área em recuperação de uma floresta natural, que possivelmente está atuando como fonte de propágulos. $\mathrm{O}$ aumento da riqueza pode estar relacionado com o aumento da diversidade funcional, influenciando, assim, a complexidade e disponibilidade de nichos em um ecossistema, o que pode contribuir para o processo de restauração, pois favorece o estabelecimento de um número maior de organismos (Cadotte et al., 2011). Valcarcel et al. (2007) encontraram que povoamentos florestais com maior diversidade de espécies e funções foram mais eficientes nos processos de reabilitação de áreas degradadas.

Das 44 espécies que foram introduzidas, apesar de a maioria ser de nativas, somente $25(56,8 \%)$ continuam existindo na área, sendo que as demais, provavelmente, não se adaptaram à região ou às condições do plantio. No entanto, não é excluída a possibilidade de que as mudas plantadas tenham atuado como facilitadoras da regeneração natural.

É possível perceber, ainda, que muitos indivíduos adultos, como Baccharis semiserrata DC. (65 indivíduos adultos) e Casearia sylvestris Sw. (47), estabeleceram-se por regeneração natural, destacando-se a importância desse processo no rápido recobrimento vegetal. Entre as espécies que foram plantadas destacaram-se em abundância entre adultos Peltophorum dubium (Spreng.) Taub. (49 indivíduos) e Mimosa scabrella Benth. (34).

No componente adulto, as famílias de maior riqueza foram Fabaceae (sete), Myrtaceae (seis) e Asteraceae (cinco). Para os jovens, as famílias de maior riqueza foram Fabaceae (11), Myrtaceae (11) e Salicaceae (quatro). Esse conjunto de famílias mais representativas, tanto dos indivíduos adultos como dos jovens, assemelham-se aos de outros inventários da vegetação arbórea realizados nas fitofisionomias de Floresta Ombrófila Densa (FOD) (Colonetti et al., 2009), Floresta Ombrófila Mista (FOM) (Higuchi et al., 2012a, 2012b) e área de ecótono entre FOD e FOM (Silva et al., 2013).

A família Myrtaceae tem sido indicada para a revegetação de áreas perturbadas (Lorenzi, 1998) 
Tabela 1. Espécies plantadas (sobreviventes ou não) e regenerantes, com o respectivo número de indivíduos, no componente arbóreo adulto (A) e jovem (J), síndrome de dispersão (SD) e guilda de regeneração (GR) em um trecho de APP (Área de Preservação Permanente) em processo de restauração no município de Pouso Redondo, SC. Table 1. Planted (survivors or not) and regenerative species, with their respective number of individuals in the adult (A) and juvenile (B) components, dispersion syndrome (SD) and regeneration guild (GR) in a patch of APP (Area of Permanent Preservation) in restoration process, in the municipality Pouso Redondo, SC.

\begin{tabular}{|c|c|c|c|c|c|}
\hline Famílias & Espécies & $\mathbf{A}$ & $\mathbf{J}$ & SD & RG \\
\hline Adoxaceae & Sambucus australis Cham. e Schltdl.* & - & - & - & - \\
\hline \multirow[t]{2}{*}{ Anacardiaceae } & Schinus molle L.* & 0 & 2 & Zoo & $\mathrm{P}$ \\
\hline & Schinus terebinthifolius Raddi $^{*}$ & 20 & 39 & Zoo & $\mathrm{P}$ \\
\hline \multirow[t]{3}{*}{ Annonaceae } & Annona cf. neosalicifolia H. Rainer* & 2 & 11 & Zoo & CEL \\
\hline & Annona rugulosa (Schltdl.) H. Rainer & 1 & 4 & Zoo & CEL \\
\hline & Annona sylvatica A. St.-Hil.* & 10 & 52 & Zoo & CEL \\
\hline \multirow[t]{3}{*}{ Apocynaceae } & Aspidosperma australe Müll. Arg.* & 0 & 1 & Ane & CEL \\
\hline & Aspidosperma tomentosum Mart. & 3 & 3 & Ane & CEL \\
\hline & Tabernaemontana catharinensis A. DC. & 3 & 5 & Zoo & CEL \\
\hline Aquifoliaceae & Ilex microdonta Reissek & 1 & 0 & Zoo & CTS \\
\hline Araucariaceae & Araucaria angustifolia (Bertol.) Kuntze & - & - & - & - \\
\hline \multirow{5}{*}{ Asteraceae } & Baccharis dracunculifolia DC. & 1 & 7 & Ane & $\mathrm{P}$ \\
\hline & Baccharis semiserrata DC. & 65 & 13 & Ane & $\mathrm{P}$ \\
\hline & Gochnatia polymorpha (Less.) Cabrera & 3 & 4 & Ane & $\mathrm{P}$ \\
\hline & Piptocarpha angustifolia Dusén & 3 & 0 & Ane & $\mathrm{P}$ \\
\hline & Vernonanthura discolor (Spreng.) H. Rob. & 1 & 0 & Ane & $\mathrm{P}$ \\
\hline \multirow[t]{5}{*}{ Bignoniaceae } & Cybistax antisyphilitica (Mart.) Mart.* & - & - & - & - \\
\hline & Handroanthus cf. impetiginosus (Mart. ex DC.) Mattos* & 0 & 1 & Ane & CEL \\
\hline & Handroanthus cf. serratifolius (Vahl) S. O. Grose* & 0 & 4 & Ane & CEL \\
\hline & Jacaranda mimosifolia D. Don.* & - & - & - & - \\
\hline & Jacaranda puberula Cham. & 3 & 3 & Ane & CEL \\
\hline Boraginaceae & Cordia trichotoma (Vell.) Arrab. ex Steud. ${ }^{*}$ & - & - & - & - \\
\hline Canellaceae & Cinnamodendron dinisii Schwacke & 0 & 4 & Zoo & CTS \\
\hline Cannabaceae & Trema micrantha (L.) Blume & 3 & 1 & Zoo & $\mathrm{P}$ \\
\hline Cunoniaceae & Lamanonia ternata Vell. & 1 & 0 & Ane & CEL \\
\hline Erythroxylaceae & Erythroxylum deciduum A. St.-Hil. & 0 & 1 & Zoo & CEL \\
\hline \multirow[t]{2}{*}{ Euphorbiaceae } & Alchornea triplinervia (Spreng.) Müll. Arg.* & 5 & 2 & $\mathrm{Au}$ & $\mathrm{P}$ \\
\hline & Sapium glandulosum (L.) Morong & 0 & 1 & $\mathrm{Au}$ & CEL \\
\hline \multirow[t]{14}{*}{ Fabaceae } & Cassia leptophylla Vogel ${ }^{*}$ & - & - & - & - \\
\hline & Dalbergia frutescens (Vell.) Britton & 0 & 1 & Ane & CEL \\
\hline & Enterolobium contortisiliquum (Vell.) Morong* & - & - & - & - \\
\hline & Inga edwalii (Harms) T. D. Penn.* & 0 & 3 & Zoo & CEL \\
\hline & Inga marginata Willd. ${ }^{\star}$ & 9 & 7 & Zoo & CEL \\
\hline & Lonchocarpus sp. & 2 & 15 & Ane & - \\
\hline & Machaerium paraguariense Hassl. & 1 & 8 & Ane & CEL \\
\hline & Mimosa scabrella Benth.* & 34 & 1 & $\mathrm{Au}$ & $\mathrm{P}$ \\
\hline & Myrocarpus frondosus Allemão* & 1 & 1 & Ane & CEL \\
\hline & Parapiptadenia rigida (Benth.) Brenan* & - & - & - & - \\
\hline & Peltophorum dubium (Spreng.) Taub.* & 49 & 8 & $\mathrm{Au}$ & $\mathrm{P}$ \\
\hline & Senna neglecta (Vogel) H.S.Irwin e Barneby & 0 & 1 & $\mathrm{Au}$ & $\mathrm{P}$ \\
\hline & Senna oblongifolia (Vogel) H. S. Irwin e Barneby* & 9 & 5 & $\mathrm{Au}$ & $\mathrm{P}$ \\
\hline & Zollernia ilicifolia (Brongn.) Vogel & 0 & 1 & Zoo & CEL \\
\hline \multirow[t]{2}{*}{ Lamiaceae } & Aegiphila sellowiana Cham. & 5 & 9 & Zoo & $\mathrm{P}$ \\
\hline & Vitex megapotamica (Spreng.) Moldenke & 0 & 3 & Zoo & CEL \\
\hline Lauraceae & Ocotea cf. silvestris Vattimo-Gil & 0 & 3 & Zoo & CEL \\
\hline
\end{tabular}

NI: não identificadas; Ane: anemocóricas; Aut: autocórica; Zoo: zoocórica; CLE: clímax exigente em luz; CTS: clímax tolerante à sombra; P: pioneira. *Espécies plantadas em 2007, sendo que as que não possuem número de indivíduos são as espécies que não foram iventariadas em 2013. 
Tabela 1. Continuação...

Tabela 1. Continued...

\begin{tabular}{|c|c|c|c|c|c|}
\hline Famílias & Espécies & A & $\mathbf{J}$ & SD & RG \\
\hline & Ocotea mandioccana A. Quinet* & - & - & - & - \\
\hline & Ocotea odorifera (Vell.) Rohwer ${ }^{*}$ & - & - & - & - \\
\hline & Ocotea porosa $(\mathrm{Mez})$ L. Barroso* & - & - & - & - \\
\hline & Ocotea puberula (Rich.) Nees & 8 & 23 & Zoo & CEL \\
\hline Lythraceae & Lafoensia vandelliana Cham. e Schltdl.* & 2 & 4 & Zoo & CEL \\
\hline Melastomataceae & Miconia sellowiana Naudin & 0 & 32 & Zoo & $\mathrm{P}$ \\
\hline \multirow[t]{2}{*}{ Meliaceae } & Cabralea canjerana (Vell.) Mart.* & 0 & 1 & Zoo & CTS \\
\hline & Cedrela fissilis Vell.* & - & - & - & - \\
\hline Monimiaceae & Mollinedia triflora (Spreng.) Tul. & 0 & 1 & Zoo & CTS \\
\hline Moraceae & Ficus luschnathiana (Miq.) Miq. & 0 & 1 & Zoo & CEL \\
\hline \multirow[t]{18}{*}{ Myrtaceae } & Acca sellowiana (O. Berg) Burret* & 0 & 2 & Zoo & $\mathrm{P}$ \\
\hline & Campomanesia $\mathrm{cf}$. reitziana D. Legrand ${ }^{*}$ & 5 & 12 & Zoo & CEL \\
\hline & Campomanesia guaviroba (DC.) Kiaersk. & 5 & 27 & Zoo & CTS \\
\hline & Campomanesia xanthocarpa O. Berg* & - & - & - & - \\
\hline & Eugenia brasiliensis Lam.* & 0 & 1 & Zoo & CEL \\
\hline & Eugenia hiemalis Cambess. & 1 & 11 & Zoo & CEL \\
\hline & Eugenia involucrata DC.* & - & - & - & - \\
\hline & Eugenia multicostata D. Legrand* & - & - & - & - \\
\hline & Eugenia pluriflora DC. & 0 & 1 & Zoo & CEL \\
\hline & Eugenia pyriformis Cambess.* & - & - & - & - \\
\hline & Eugenia uniflora L. $^{*}$ & - & - & - & - \\
\hline & Myrcia hatschbachii D. Legrand & 1 & 8 & Zoo & CTS \\
\hline & Myrcia hebepetala DC.* & 0 & 4 & Zoo & CEL \\
\hline & Myrcia oblongata DC. & 0 & 1 & Zoo & $\mathrm{P}$ \\
\hline & Myrcianthes pungens (O. Berg) D. Legrand* & - & - & - & - \\
\hline & Plinia trunciflora (O.Berg) Kausel ${ }^{\star}$ & - & - & - & - \\
\hline & Psidium cattleianum Sabine ${ }^{*}$ & 2 & 10 & Zoo & CEL \\
\hline & Psidium guajava L. & 4 & 11 & Zoo & CEL \\
\hline Podocarpaceae & Podocarpus lambertii Klotzsch ex Endl.* & 0 & 3 & Zoo & CEL \\
\hline \multirow[t]{2}{*}{ Primulaceae } & Myrsine coriacea (Sw.) Roem. e Schult.* & 16 & 26 & Zoo & CEL \\
\hline & Myrsine lorentziana (Mez) Arechav.* & 0 & 2 & Zoo & CEL \\
\hline \multirow[t]{2}{*}{ Rubiaceae } & Psychotria vellosiana Benth. & 0 & 1 & Zoo & CEL \\
\hline & Randia ferox DC. & 0 & 4 & Zoo & CTS \\
\hline \multirow[t]{3}{*}{ Rutaceae } & Citrus sp.1 & 0 & 1 & Zoo & - \\
\hline & Citrus sp. 2 & 1 & 0 & Zoo & - \\
\hline & Zanthoxylum rhoifolium Lam. & 6 & 11 & Zoo & CEL \\
\hline \multirow[t]{4}{*}{ Salicaceae } & Casearia decandra Jacq. & 0 & 1 & Zoo & CTS \\
\hline & Casearia obliqua Spreng. & 0 & 1 & Zoo & CTS \\
\hline & Casearia sylvestris $\mathrm{Sw}$. & 47 & 75 & Zoo & CEL \\
\hline & Xylosma ciliatifolia (Clos) Eichler & 0 & 5 & Zoo & CEL \\
\hline \multirow[t]{3}{*}{ Sapindaceae } & Allophylus edulis (A. St.-Hil., Cambess. e A. Juss.) Radlk. & 0 & 22 & Zoo & CEL \\
\hline & Cupania vernalis Cambess. & 0 & 9 & Zoo & CEL \\
\hline & Matayba juglandifolia Radlk & 3 & 4 & Zoo & CEL \\
\hline \multirow[t]{3}{*}{ Solanaceae } & Brunfelsia cuneifolia J. A. Schmidt & 0 & 1 & Zoo & CTS \\
\hline & Solanaceae sp.1 & 2 & 2 & Zoo & - \\
\hline & Solanum mauritianum Scop. & 16 & 6 & Zoo & $\mathrm{P}$ \\
\hline Verbenaceae & Citharexylum myrianthum Cham. & 1 & 0 & Zoo & $\mathrm{P}$ \\
\hline NI & NI & 11 & 4 & - & - \\
\hline Total & & 366 & 552 & & \\
\hline
\end{tabular}

NI: não identificadas; Ane: anemocóricas; Aut: autocórica; Zoo: zoocórica; CLE: clímax exigente em luz; CTS: clímax tolerante à sombra; P: pioneira. ${ }^{*}$ Espécies plantadas em 2007, sendo que as que não possuem número de indivíduos são as espécies que não foram iventariadas em 2013. 
devido a suas importantes funções, como a atração de insetos polinizadores, especialmente abelhas, e da fauna dispersora de frutos, principalmente aves, roedores e macacos (Barroso et al., 1999). Em relação à Fabaceae, sua riqueza específica pode ser devida ao rápido desenvolvimento e capacidade de adaptação de espécies da família em áreas abertas, principalmente de Mimosa scabrella Benth e Peltophorum dubium (Spreng.) Taub. A grande representatividade de Fabaceae e a reconhecida importância das espécies dessa família para o rápido estabelecimento, incorporação de fitomassa e fixação biológica de nitrogênio (Almeida et al., 2009) apontam o grande potencial da família para a recuperação de áreas degradadas.

Os gêneros de maior riqueza de espécies adultas foram Annona (três), Baccharis (duas), Campomanesia (duas) e Psidium (duas), e os dos jovens foram Annona (três), Casearia (três), Eugenia (três) e Myrcia (três). A elevada riqueza de gêneros como Baccharis, por exemplo, indica o caráter inicial do processo de restauração, pois eles apresentam, em geral, espécies que preferem áreas abertas ou florestas alteradas, onde existe maior incidência de luz. A presença de duas espécies exóticas do gênero Citrus e de Psidium guajava L., que não foram cultivadas, pode ser explicada pela ocorrência de forma subespontânea, provavelmente com a semente dispersa pela avifauna.

O índice de diversidade de Shannon para os indivíduos adultos $\left(\mathrm{H}^{\prime}=2,91\right)$ pode ser considerado baixo quando comparado com outros estudos realizados em florestas secundárias de Santa Catarina, como o de Silva et al. (2012), que encontraram H' = 3,6 em um fragmento de FOM, e o de Colonetti et al. (2009), que obtiveram $\mathrm{H}^{\prime}=3,23 \mathrm{em}$ uma área de FOD. Em ambos os casos, as florestas eram secundárias, porém, em avançado estágio de sucessão, diferente da área de estudo, que se encontra em estágio inicial.

Em relação aos indivíduos jovens, o índice de diversidade indicou alta diversidade de espécies $\left(H^{\prime}=3,5\right)$ se comparado a estudos realizados com esse componente, como o de Narvaes et al. (2005), que encontraram $\mathrm{H}^{\prime}=2,22$ em FOM (com CAP - circunferência medida a altura do peito - entre 3 a $30 \mathrm{~cm}$ ). A alta diversidade dos indivíduos jovens sugere que o processo de restauração da área esteja ocorrendo de forma adequada, uma vez que em estágios iniciais de sucessão espera-se o aumento da diversidade a partir do estabelecimento de novas espécies na área. O fato de a área ter sido protegida e isolada de distúrbios contribui para o incremento da diversidade relativa do componente de jovens, o que demonstra que esses tipos de medidas conservacionistas são de grande importância para a restauração ecológica quando se tem a presença de fontes de propágulos próximas, como é o caso do presente estudo. Os índices de Pielou foram elevados tanto para adultos $(J=0,78)$ quanto para jovens $(J=0,83)$, indicando baixa dominância ecológica e distribuição mais uniforme de indivíduos entre as espécies.

Em se tratando da síndrome de dispersão, a maioria das 40 espécies dos indivíduos adultos foi classificada como zoocórica $(62,5 \%)$, seguidas pelas anemocóricas (27,5\%) e autocóricas (10,0\%) (Tabela 2). As 67 espécies dos indivíduos jovens seguiram o mesmo padrão, sendo $73,1 \%$ zoocóricas, $17,9 \%$ anemocóricas e 9,0\% autocóricas. Dentre as famílias de maior riqueza destacadas, Myrtaceae apresenta diásporos dispersos por animais, enquanto que Fabaceae apresentou os três tipos de dispersão. A síndrome de dispersão por zoocoria apresentou a maior proporção de espécies $(71,2 \%)$, demonstrando a importância da fauna na manutenção das espécies arbóreas florestais e, consequentemente,

Tabela 2. Número de espécies classificadas segundo sua síndrome de dispersão e guilda de regeneração encontradas em um trecho de APP (Área de Preservação Permanente) em processo de restauração no município de Pouso Redondo, SC.

Table 2. Number of species classified according to their dispersion syndrome and regeneration guild, found in a patch of APP (Area of Permanent Preservation) in restoration process, in the municipality of Pouso Redondo, SC.

\begin{tabular}{cccc}
$\begin{array}{c}\text { Síndromes } \\
\text { de dispersão }\end{array}$ & Adultos & Jovens & Total \\
\hline Zoocórica & $25(62,5 \%)$ & $49(73,1 \%)$ & $52(71,2 \%)$ \\
\hline Anemocórica & $11(27,5 \%)$ & $12(17,9 \%)$ & $15(20,6 \%)$ \\
\hline Autocórica & $4(10,0 \%)$ & $6(9,0 \%)$ & $6(8,2 \%)$ \\
Total & $40(100,0 \%)$ & $67(100,0 \%)$ & $73(100,0 \%)$ \\
\hline $\begin{array}{c}\text { Guildas de } \\
\text { Regeneração }\end{array}$ & Adultos & Jovens & Total \\
\hline P & $14(35,0 \%)$ & $16(23,9 \%)$ & $19(26,0 \%)$ \\
CEL & $20(50,0 \%)$ & $39(58,2 \%)$ & $40(54,8 \%)$ \\
CTS & $3(7,5 \%)$ & $9(13,4 \%)$ & $10(13,7 \%)$ \\
\hline NC & $3(7,5 \%)$ & $3(4,5 \%)$ & $4(5,5 \%)$ \\
\hline Total & $40(100,0 \%)$ & $67(100,0 \%)$ & $73(100,0 \%)$ \\
\hline
\end{tabular}

P: pioneira; CEL: clímax exigente em luz; CTS: clímax tolerante à sombra; NC: não classificadas devido à espécie estar identificada somente em nível de gênero ou família. 
no equilíbrio dos ecossistemas. Para Reis et al. (1999), o comportamento dos animais ao transportarem as sementes e dispersá-las é, na restauração de áreas degradadas, um auxílio fundamental.

Em relação aos grupos sucessionais, o grupo das espécies clímax exigentes em luz (CEL) foi o que mais se destacou, com 50,0\% das espécies adultas e $58,2 \%$ das jovens. As pioneiras ocuparam a segunda colocação, com $35,0 \%$ das adultas e $23,9 \%$ das jovens, enquanto que as tolerantes à sombra (CTS) foram $7,5 \%$ das adultas e $13,4 \%$ das regenerantes. A riqueza de espécies pioneiras é característica de vegetação em estágio inicial de regeneração e a maior proporção dessas nas espécies adultas indica que está havendo uma mudança sucessional na área, ou seja, uma substituição das pioneiras por CEL e CTS, de maior proporção nas jovens. Considerando-se que as espécies jovens irão substituir, no futuro, as adultas, isso demonstra um processo de sucessão ecológica com aumento das CEL e CTS.

\section{CONCLUSÕES}

A análise dos componentes adultos e jovens da vegetação arbórea permitiu concluir que, seis anos após o plantio inicial de mudas e isolamento da área contra distúrbios, o mecanismo de regeneração natural desempenhou importante função no processo de restauração florestal. Ficou evidenciada uma tendência de aumento da riqueza e de substituição de espécies iniciais por tardias no processo de sucessão. Além disso, destaca-se a importância da fauna no processo de restauração, uma vez que zoocoria foi a síndrome de dispersão mais relevante, tanto para adultos quanto para jovens.

\section{STATUS DA SUBMISSÃO}

Recebido: 19 mar., 2014

Aceito: 14 ago., 2014

\section{AUTOR(ES) PARA CORRESPONDÊNCIA}

\section{Pedro Higuchi}

Departamento de Engenharia Florestal, Universidade do Estado de Santa Catarina UDESC, CEP 88520-000, Lages, SC, Brasil e-mail: higuchip@gmail.com

\section{REFERENNCIAS}

Almeida LGF, Moreira GGDLB, Rodrigues CC, Oliveira FL, Favero C. Levantamento de espécies arbóreas de Matas Ciliares como subsídio a implantação de sistemas agroflorestais em áreas de agricultura familiar no Vale do Mucuri, Minas Gerais, Brasil. Revista Brasileira de Agroecologia 2009; 4(2): 1999-2000.

Almeida ROPO, Sánchez LE. Revegetação de áreas de mineração: critérios de monitoramento e avaliação do desempenho. Revista Árvore 2005; 29(1): 47-54. http:// dx.doi.org/10.1590/S0100-67622005000100006.

Almeida SR, Watzlawick LF, Myszka E, Valerio AF. Florística e síndromes de dispersão de um remanescente de Floresta Ombrófila Mista em sistema faxinal. Revista Ambiência 2008; 4(2): 289-297.

Alvarenga AP, Botelho SA, Pereira IM. Avaliação da regeneração natural na recomposição de matas ciliares em nascentes na região sul de Minas Gerais. Cerne 2006; 12(4): 360-372.

Angiosperm Phylogeny Group - APG. An update of the Angiosperm Phylogeny Group classification for the orders and families of flowering plants: APG III. Botanical Journal of the Linnean Society 2009; 161(2): 105-121. http://dx.doi. org/10.1111/j.1095-8339.2009.00996.x.

Araujo MM, Longhi SI, Barros PLC, Brena DA. Caracterização da chuva de sementes, banco de sementes do solo e banco de plântulas em Floresta Estacional Decidual ripária Cachoeira do Sul, RS, Brasil. Scientia Florestalis 2004; 66(50): 128-141.

Attanasio CL, Rodrigues RR, Gandolfi S, Nave AG. Adequação Ambiental de propriedades rurais, recuperação de áreas degradadas, restauração de matas ciliares. São Paulo: ESALQ; 2006. [cited 2014 Jan 15]. Available from: http:// www.sigam.ambiente.sp.gov.br/sigam2/Repositorio/222/ Documentos/Gestao\%20Projetos/20061_ap_LERF.pdf.

Barroso GM, Morin MP, Peixoto AL, Ichaso CLF. Frutose sementes: morfologia aplicada à sistemática de dicotiledôneas. Viçosa: Editora UFV; 1999.

Brower JE, Zar JH. Field and laboratory methods for general ecology. Duduque: W.M.C. Brow Publishers; 1984.

Budke JC, Athayde EA, Giehl ELH, Zachia RA, Eisinger SM. Composição florística e estratégias de dispersão de espécies lenhosas em uma floresta ribeirinha, arroio Passo das Tropas, Santa Maria, RS, Brasil. Iheringia, Série Botânica 2005; 60(1): 17-24.

Cadotte MW, Carscadden K, Mirotchnick N. Beyond species: functional diversity and the maintenance of ecological processes and services. Journal of Applied Ecology 2011; 48(5): 1079-1087. http://dx.doi.org/10.1111/j.13652664.2011.02048.x.

Colonetti S, Citadini-Zanette V, Martins R, Santos R, Rocha E, Jarenkow JE. Florística e estrutura fitossociológica em Floresta Ombrófila Densa Submontana na barragem do 
rio São Bento, Siderópolis, Estado de Santa Catarina. Acta Scientiarum. Biological Sciences 2009; 31(4): 397-405.

Durigan G, Silveira ÉR. Recomposição da mata ciliar em domínio de Cerrado, Assis, SP, Brasil. Scientia Forestalis 1999; 56(50): 135-144.

Figueiredo AG. Avaliação da recuperação de área degradada pela Mineração de argila através do plantio de Mimosa scabrella Benth. (Fabaceae), sob duas técnicas de preparação do solo, Doutor Pedrinho, SC [dissertação]. Blumenau: Departamento de Engenharia Ambiental, Universidade Regional de Blumenau; 2005.

Giehl ELH, Athayde EA, Budke JC, Gesing JPA, Einsiger SM, Canto-Dorow TS. Espectro e distribuição vertical das estratégias de dispersão de diásporos do componente arbóreo em uma floresta estacional no sul do Brasil. Acta Botanica Brasilica 2007; 21(1): 137-145. http://dx.doi. org/10.1590/S0102-33062007000100013.

Higuchi P, Silva AC, Ferreira TS, Souza ST, Gomes JP, Silva KM, et al. Influência de variáveis ambientais sobre o padrão estrutural e florístico do componente arbóreo, em um fragmento de Floresta Ombrófila Mista Montana em Lages, SC. Ciência Florestal 2012a; 22(1): 79-90.

Higuchi P, Silva AC, Ferreira TS, Souza ST, Gomes JP, Silva $\mathrm{KM}$, et al. Floristic composition and phytogeography of the tree component of Araucaria Forest fragments in southern Brazil. Brazilian Journal of Botany 2012b; 35(2): 145-157. http://dx.doi.org/10.1590/S1806-99592012000200004.

Hijmans RJ, Cameron SE, Parra JL, Jones PG, Jarvis A. Very high resolution interpolated climate surfaces for global land areas. International Journal of Climatology 2005; 25(15): 1965-1978. http://dx.doi.org/10.1002/joc.1276.

Instituto Brasileiro de Geografia e Estatística - IBGE. Manual técnico da vegetação brasileira. Rio de Janeiro; 2012.

Kageyama PY, Gandara FB. Resultados do programa de restauração com espécies arbóreas nativas do convênio ESALQ/USP e CESP. In: Galvão APM, Porfírio-da-Silva $\mathrm{V}$, editores. Restauração florestal: fundamentos e estudos de caso. Colombo: Embrapa; 2005.

Leyser G, Viniski M, Donida AL, Zanin EM, Budke JC. Espectro de dispersão em um fragmento de transição entre Floresta Ombrófila Mista e Floresta Estacional na região do Alto Uruguai, Rio Grande do Sul, Brasil. Pesquisas, Série Botânica 2009; (60): 355-366.

Leyser G, Zanin EM, Budke JC, Mélo MA, Henke-Oliveira C. Regeneração de espécies arbóreas e relações com o componente adulto em uma floresta estacional no vale do rio Uruguai, Brasil. Acta Botanica Brasilica 2012; 26(1): 7483. http://dx.doi.org/10.1590/S0102-33062012000100009.

Lindenmaier DS, Budke JC. Florística, diversidade e distribuição espacial das espécies arbóreas em uma floresta estacional na bacia do rio Jacuí, Sul do Brasil. Pesquisas, Série Botânica, 2006; (57): 193-216.
Lorenzi H. Árvores brasileiras: manual de identificação e cultivo de plantas arbóreas nativas do Brasil. 2. ed. Nova Odessa: Plantarum; 1998. vol. 2.

Narvaes IS, Brena DA, Longhi SJ. Estrutura da regeneração natural em Floresta Ombrófila Mista na Floresta Nacional de São Francisco de Paula, RS. Ciência Florestal 2005; 15(4): 331-342.

Negrini M, Aguiar MD, Vieira CT, Silva AC, Higuchi P. Dispersão, distribuição espacial e estratificação vertical da comunidade arbórea em um fragmento florestal no Planalto Catarinense. Revista Árvore 2012; 36(5): 919-930. http://dx.doi.org/10.1590/S0100-67622012000500014.

Oliveira Filho AT, Vilela EA, Carvalho DA, Gavilanes ML. Effects of soils and topography on the distribution of tree species in a tropical riverine forest in south-eastern Brazil. Journal of Tropical Ecology 1994; 10(4): 483-508. http://dx.doi.org/10.1017/S0266467400008178.

R Development Core Team. $R$ : a language and environment for statistical computing [cited 2014 Jan 15]. Available from: http://www.R-project.org.

Reis A, Zambonin RM, Nakazono EM. Recuperação de áreas florestais degradadas utilizando a sucessão e as interações planta-animal. São Paulo: Instituto Florestal; 1999. Série Cadernos da Reserva da Biosfera da Mata Atlântica. n. 3, Caderno 14.

Reis A, Tres DR, Scariot EC. Restauração na Floresta Ombrofila Mista através da sucessão natural. Pesquisa Florestal Brasileira 2007; (55): 67-73.

Rodrigues RR, Gandolfi S, Nave AG, Attanasio CM. Atividade de adequação ambiental e restauração florestal do LERF/ESALQ/USP. Pesquisa Florestal Brasileira 2007; (55): 7-21.

Rondon Neto RM, Watzlawick LF, Caldeira MVW. Diversidade florística e síndromes de dispersão de diásporos das espécies arbóreas de um fragmento de Floresta Ombrófila Mista. Revista Ciências Exatas e Naturais 2001; 3(2): 167-175.

Santos JF, Valcarcel R. Avaliação Florística do Estrato Regenerante de Reflorestamentos em área Reabilitada na Mata Atlântica. Floresta e Ambiente 2011; 18(4): 390-401. http://dx.doi.org/10.4322/floram.2011.058.

Silva AC, Higuchi P, Aguiar MD, Negrini M, Fert Neto JF, Hess AF. Relações florísticas e fitossociologia de uma Floresta Ombrófila Mista Montana secundária em Lages, Santa Catarina. Ciência Florestal 2012; 22(1): 193-206.

Silva AC, Higuchi P, Negrini M, Grudtner A, Zech DF. Caracterização fitossociológica e fitogeográfica de um trecho de floresta ciliar em Alfredo Wagner, SC, como subsídio para restauração ecológica. Ciência Florestal 2013; 23(4): 579-593.

Swaine MD, Whitmore TC. On the definition of ecological species groups in tropical rain forest. Vegetatio 1988; 75(1-2): 81-86. http://dx.doi.org/10.1007/BF00044629. 
Valcarcel R, D’Altério CF. Medidas fisico-biológicas de recuperação de áreas degradadas: avaliação das modificações edáficas e fitossociológicas. Floresta e Ambiente 1998; 5(1): 68-88.

Valcarcel R, Valente FDW, Morokawa MJ, Cunha Neto FV, Pereira CR. Avaliação da biomassa de raízes finas em áreas de empréstimo submetida a diferentes composições de espécies. Revista Árvore 2007; 31(5): 923-930. http:// dx.doi.org/10.1590/S0100-67622007000500016.

Van der Pijl L. Principles of dispersal in higher plants. Berlim: Springer-Verlag; 1982. http://dx.doi.org/10.1007/978-3642-87925-8. 